Cite this: J. Mater. Chem. A, 2013, 1, 11641

Received 9th July 2013

Accepted 26th July 2013

DOI: $10.1039 / c 3 t a 12660 a$

www.rsc.org/MaterialsA

\section{A double-shelled yolk-like structure as an ideal magnetic support of tiny gold nanoparticles for nitrophenol reduction $\uparrow$}

\author{
Tao Zeng, ${ }^{\text {a }}$ Xiaole Zhang, ${ }^{\text {ab }}$ Saihua Wang, ${ }^{\mathrm{c}}$ Yurong Ma, ${ }^{\mathrm{a}}$ Hongyun $\mathrm{Niu}^{\mathrm{a}}$ and Yaqi Cai ${ }^{\star a}$ \\ A facile method is proposed to fabricate yolk-shell microspheres consisting of a movable silica core, a \\ multifunctional double-layered shell, and plenty of tiny gold nanoparticles (Au NPs, $\sim 2 \mathrm{~nm}$ ) confined \\ within the interior cavity and the mesoporous shell. The presented strategy involves the one-step \\ coating of a $\mathrm{Fe}_{3} \mathrm{O}_{4}$ /carbon double-layered shell, the partial etching of the silica cores and the in situ \\ immobilization of $\mathrm{Au}$ NPs. The inner $\mathrm{Fe}_{3} \mathrm{O}_{4}$ layer of the double-layered shell endows the composites \\ with superparamagnetism and thereby simplifies the introduction procedure of a magnetic component. \\ The outer carbon layer not only protects the $\mathrm{Fe}_{3} \mathrm{O}_{4}$ layer from outside harsh conditions but also \\ provides additional adsorption sites for Au NPs besides the interior space. The large number of catalytic \\ active sites together with the advantages of the yolk-shell architecture make the nanocomposite a \\ perfect catalyst for the reduction of 4-nitrophenol to 4-aminophenol in the presence of $\mathrm{NaBH}_{4}$ \\ (TOF value is $17.4 \mathrm{~min}^{-1}$ ). Moreover, the synthesized catalyst can be easily recovered and reused for at \\ least nine cycles due to its magnetically separable feature and good stability. These confirm that the as- \\ prepared yolk-like nanocomposites are promising candidates for catalytic application.
}

\section{Introduction}

Yolk-shell or rattle-type nanostructures that consist of a core encapsulated in a hollow capsule with a mesoporous shell are emerging as an interesting family of complex nanoarchitectures. These systems have attracted considerable attention over the past few years in a variety of applications including catalysis, storage, and drug delivery due to their unique properties, such as large surface area, multi-functionality and excellent loading capacity. ${ }^{1}$ The yolk-shell structures can hinder the aggregation of the neighbouring cores and protect the core from the outside environment while allowing the diffusion of small active molecules in and out of the shell, which endow the materials with promising applications as nanoreactors for catalysis. In the past decade, many yolk-shell nanomaterials with a movable metal core embedded in a hollow mesoporous sphere (such as Au@polymer, Au@carbon, $\mathrm{Au} @$ silica, etc.) have been designed and successfully applied for catalysis. ${ }^{2}$ Among these studies, multiple coating of the preformed metal nanoparticles followed by removing the middle

${ }^{a}$ State Key Laboratory of Environmental Chemistry and Ecotoxicology, Research Center for Eco-Environmental Sciences, Chinese Academy of Sciences, P.O. Box 2871, Beijing 100085, China. E-mail: caiyaqi@rcees.ac.cn; Tel: +86-10-6284-9182

${ }^{b}$ College of life science, Hebei United University, Tangshan, Hebei, 06300o, China

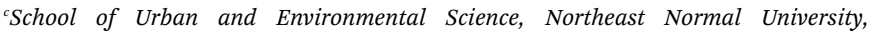
Changchun, Jilin, 130024, China

$\dagger$ Electronic supplementary information (ESI) available. See DOI: $10.1039 / \mathrm{c} 3 \mathrm{ta} 12660 \mathrm{a}$ layer is a common strategy. ${ }^{3}$ However, the sizes of the metal cores were somewhat big, resulting in a relatively low catalytic performance due to the low surface area per unit mass. It remains a challenge to generate extra fine metal nanoparticles (smaller than $10 \mathrm{~nm}$ ) in the hollow mesoporous capsule to achieve a higher catalytic efficiency.

Most recently, selectively hosting some tiny active catalytic nanoparticles in the void space of yolk-shell materials instead of enveloping a sole metal core inside the hollow capsule was proposed as an alternative tactic to meet this goal. ${ }^{4}$ For instance, Yang reported $\mathrm{SiO}_{2} @$ poly(ethyleneglycol dimethacrylate) yolk-shell microspheres loaded with small gold nanoparticles in the void space which exhibited high catalytic efficiency for reduction of 4-nitrophenol. ${ }^{5}$ Qiao and coworkers demonstrated yolk-shell nanoparticles consisting of a silica core and an outer organosilica shell with metal nanoparticles encapsulated in the interstice between the core and the shell for selective oxidation of alcohol. ${ }^{6}$ Although some progress in the preparation of a small metal nanoparticle loaded yolkshell catalyst has been achieved, the materials obtained previously are mostly composed of a single-layer shell, which presents low compositional diversity and less functionality. The design of a high-efficiency yolk-shell catalyst having multiple shells with desirable components, unique structure, and diverse functionality is still highly significant. Furthermore, most of the studies focus on the synthesis of desired catalytically active cores and rare work succeeds in producing active catalysis sites on the shell. ${ }^{2 a, 7}$ If small active metal 
nanoparticles could be located both inside the void space and on the shell and the structural advantage of yolk-shell materials as a catalyst support remained simultaneously, an increasing loading amount of active catalyst would be undoubtedly achieved to improve the catalytic performance. Therefore, the design of shells that not only have functions similar to those of conventional shells but also can be used as a catalyst support would help to fabricate novel and high-efficiency yolk-shell catalysts. Another problem worthy of attention is the requirement of tedious filtration or centrifugation during the recycling of the yolk-shell catalysts synthesized in previous reports, and this greatly limits their practical applicability. ${ }^{7 a, 8}$ In this regard, the introduction of superparamagnetic property into the yolk-shell catalyst for convenient magnetic separation could be a promising solution. ${ }^{9}$ However, until now, only a few yolk-like nanomaterials with a superparamagnetic core have been synthesized by some relatively complex approaches and reports on simple methods to prepare new-style magnetic yolk-shell catalysts are scarce. ${ }^{\mathbf{1 0}}$ Hence, it is necessary to develop a novel and facile strategy for fabricating multifunctional yolk-shell catalysts with unique framework, magnetically recyclable feature, and excellent catalytic performance. Recently, our group reported an interesting rattle-type microsphere with a magnetic-carbon doublelayered shell for enhanced extraction of organic targets. ${ }^{\mathbf{1 1}}$ The unique yolk-double shell framework and the superparamagnetic character of the material give it potential for use as an ideal magnetic support for catalytically active NPs.

Inspired by this supposal, we thereby prepare yolk-double shell microspheres as a support for loading tiny gold nanoparticles in both the void space and the outer shell $\left(\mathrm{SiO}_{2} @\right.$ $\mathrm{Fe}_{3} \mathrm{O}_{4} / \mathrm{C} @ \mathrm{Au}$ ) in the present work. The nanostructure is obtained by a silica templating method in which a $\mathrm{Fe}_{3} \mathrm{O}_{4} / \mathrm{C}$ double-layered shell is first coated on the surface of $\mathrm{a} \mathrm{SiO}_{2}$ core through a one-step hydrothermal route followed by the partial etching of the silica core to form a typical yolk-shell structure (Scheme 1). The porous shell, which is composed of an inner $\mathrm{Fe}_{3} \mathrm{O}_{4}$ layer and an outer carbon layer, plays a key role in this system. The magnetic $\mathrm{Fe}_{3} \mathrm{O}_{4}$ layer enables the convenient isolation and recycling of the composite by employing an external magnetic field and the outer carbon layer can provide additional adsorption sites for the attachment of Au NPs and protect the $\mathrm{Fe}_{3} \mathrm{O}_{4}$ layer from outside harsh conditions as well.

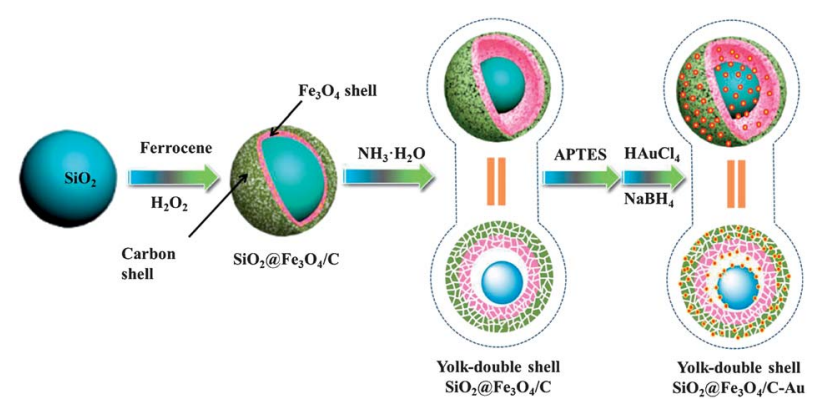

Scheme 1 Synthetic strategy of a yolk-double shell $\mathrm{SiO}_{2} @ \mathrm{Fe}_{3} \mathrm{O}_{4} / \mathrm{C} @ \mathrm{Au}$ microsphere.
Interestingly, the magnetic component here is introduced within one step as a shell rather than a big core, which can avoid the ferromagnetism of the big magnetic core and simplify the synthesis procedure evidently. ${ }^{12}$ After being decorated with tiny Au NPs both within the shell and the void space, the yolk-shell materials are employed as nanocatalysts for the reduction of 4nitrophenol with $\mathrm{NaBH}_{4}$. To the best of our knowledge, this is the first report on the synthesis of magnetically double-shelled yolk-shell microspheres with tiny noble NPs immobilized in the shell and cavity for catalysis.

\section{Experimental}

\section{Chemicals}

HPLC-grade acetone, toluene, and methanol were supplied by Fisher Scientific (Fair Lawn, NJ). Tetraethyl orthosilicate (TEOS) was obtained from Acros Organics (Morris Plains, NJ). Ammonia aqueous solution $(25 \%$, w/w) was from Alfa Aesar (Ward Hill, MA). 3-Aminopropyl-triethoxysilane (APTES) was obtained from Sigma-Aldrich (St. Louis, MO). Ferrocene, hydrogen peroxide $\left(\mathrm{H}_{2} \mathrm{O}_{2}\right)$, anhydrous ethanol, hydrogen tetrachloroaurate hydrate $\left(\mathrm{HAuCl}_{4} \cdot 4 \mathrm{H}_{2} \mathrm{O}\right)$, 4-nitrophenol, and sodium hydroxide $\left(\mathrm{NaBH}_{4}\right)$ were guarantee-grade reagents from Beijing Chemicals Co. Ltd. (Beijing, China). All chemicals were used as received without any further purification. Ultrapure water was prepared in the laboratory using a Milli-Q SP reagent water system from Millipore (Milford, MA).

\section{Synthesis of yolk-double shell $\mathrm{SiO}_{2} / \mathrm{Fe}_{3} \mathrm{O}_{4} / \mathrm{C}$ microspheres}

Monodispersed $\mathrm{SiO}_{2}$ nanoparticles were first synthesized through the Stöber method. ${ }^{\mathbf{1 3}}$ The obtained silica microspheres were coated with a double layered shell via a hydrothermal approach. ${ }^{14}$ Briefly, silica microspheres (100 mg) and ferrocene (200 $\mathrm{mg})$ were dispersed in acetone $(65 \mathrm{~mL})$ with ultrasonic treatment for $30 \mathrm{~min}$. Next, $2 \mathrm{~mL} \mathrm{H}_{2} \mathrm{O}_{2}$ was added dropwise into the mixture followed by vigorous stirring for $90 \mathrm{~min}$. After that, the solution was transferred into a Teflon-lined stainless-steel autoclave and sealed to heat at $210{ }^{\circ} \mathrm{C}$ for $48 \mathrm{~h}$. When the autoclave was cooled to room temperature, the obtained product was washed with acetone and ethanol three times respectively, and then dried under vacuum for $12 \mathrm{~h}$. To prepare rattle-type $\mathrm{SiO}_{2} / \mathrm{Fe}_{3} \mathrm{O}_{4} / \mathrm{C}$ composites, $120 \mathrm{mg}$ of the above coreshell $\mathrm{SiO}_{2} / \mathrm{Fe}_{3} \mathrm{O}_{4} / \mathrm{C}$ microspheres were dispersed in $60 \mathrm{~mL}$ of ultrapure water containing $0.1 \mathrm{~mL}$ of aqueous ammonia. After a $30 \mathrm{~min}$ stirring period, the resultant product was transferred into a Teflon-lined stainless-steel autoclave again and heated to $150{ }^{\circ} \mathrm{C}$ for $6 \mathrm{~h}$. Finally, the product was collected with the help of a magnet, and then washed with ultrapure water and methanol several times.

\section{Synthesis of yolk-double shell $\mathrm{SiO}_{2} @ \mathrm{Fe}_{3} \mathrm{O}_{4} / \mathrm{C} @ A u$ microspheres}

The as-prepared yolk-shell $\mathrm{SiO}_{2} @ \mathrm{Fe}_{3} \mathrm{O}_{4} / \mathrm{C}$ composites were first mixed with $40 \mathrm{~mL}$ of anhydrous toluene and $0.8 \mathrm{~mL}$ of APTES and heated to $120{ }^{\circ} \mathrm{C}$ for $12 \mathrm{~h}$ to functionalize the composites with amino groups. The amino modified 
microspheres were washed with ethanol and dispersed in deionized water. Then, the $\mathrm{SiO}_{2} @ \mathrm{Fe}_{3} \mathrm{O}_{4} / \mathrm{C} @ \mathrm{Au}$ composites were synthesized through an aqueous impregnation method. In a typical procedure, $4 \mathrm{~mL}$ of $\mathrm{HAuCl}_{4}$ aqueous solution $\left(4.1 \mathrm{mg} \mathrm{mL} \mathrm{mL}^{-1}\right)$ was added into $150 \mathrm{~mL}$ of amino modified yolkshell $\mathrm{SiO}_{2} @ \mathrm{Fe}_{3} \mathrm{O}_{4} / \mathrm{C}$ suspension and then stirred at room temperature overnight. After that, $2.5 \mathrm{~mL}$ of fresh $\mathrm{NaBH}_{4}$ (0.1 M) was added into the mixture and the reaction was allowed to proceed for another $2 \mathrm{~h}$ with stirring. The products were magnetically separated from the solution and rinsed with deionized water and ethanol three times to remove the free $\mathrm{Au}$ NPs, followed by drying under vacuum for $12 \mathrm{~h}$.

\section{Catalytic reduction of 4-nitrophenol}

The reduction of 4-nitrophenol in the presence of $\mathrm{NaBH}_{4}$ was carried out to investigate the catalytic activity and reusability of the yolk-like $\mathrm{SiO}_{2} @ \mathrm{Fe}_{3} \mathrm{O}_{4} / \mathrm{C} @ A u$ catalyst. Typically, $2 \mathrm{~mL}$ of deionized water, $1.0 \mathrm{~mL}$ of fresh $\mathrm{NaBH}_{4}(0.2 \mathrm{M})$, and $0.1 \mathrm{~mL} 4$ nitrophenol solution $\left(5 \times 10^{-3} \mathrm{M}\right)$ were added into a quartz cuvette in sequence, followed by the addition of $0.05 \mathrm{~mL}$ of catalyst $\left(1 \mathrm{mg} \mathrm{mL}^{-1}\right)$ to the mixture. The reaction progress was monitored by UV-vis spectroscopy at a certain time interval to obtain the successive information. To determine the reusability, the amounts of 4-nitrophenol and catalyst were increased to 20 times and 10 times of the above typical reduction, respectively. When the reduction process was completed, the catalysts were isolated by an external magnet and reused in the next cycle. The $\mathrm{Au}$ content of the catalyst was measured by inductively coupled plasma mass spectrometry (ICP-MS, Agilent, CA).

\section{Characterization}

The size and morphology of the synthesized materials were measured using a Hitachi S-5500 field-emission scanning electron microscope (FE-SEM, Tokyo, Japan), JEOL JEM-2010 highresolution transmission electron microscope (HRTEM, Kyoto, Japan), and Tecnai G2 F20 HRTEM with an energy dispersive X-ray spectrometer (HRTEM-EDX, FEI, Netherlands). X-Ray diffraction studies (XRD, PANalytical X'Pert diffractometer, Almelo, Netherlands) were performed by using a monochromatized X-ray beam with nickel-filtered $\mathrm{Cu} \mathrm{K} \alpha$ radiation with $0.4^{\circ} \min ^{-1}$ scan rate. X-Ray photoelectron spectroscopy (XPS) measurements were conducted by using a Thermo Scientific ESCA-Lab-200i-XL spectrometer (Waltham, MA) with monochromatic $\mathrm{Al} \mathrm{K} \alpha$ radiation $(1486.6 \mathrm{eV})$. FTIR spectra were taken in $\mathrm{KBr}$ pressed pellets on a Nicolet Thermo NEXUS 670 Infrared Fourier Transform Spectrometer (Waltham, MA). The magnetization curves of the products were obtained with an LDJ9600 vibrating sample magnetometer (VSM, Troy, MI). Nitrogen sorption isotherms were obtained at $77 \mathrm{~K}$ with a Quadrasorb $^{\text {TM }}$ SI Four Station Surface Area Analyzer and Pore Size Analyzer (Quantachrome Instruments, Boynton Beach, FL). Before measurements, the samples were degassed in a vacuum at $300{ }^{\circ} \mathrm{C}$ for at least $6 \mathrm{~h}$. The Brunauer-Emmett-Teller (BET) method was utilized to calculate the specific surface areas using adsorption data in a relative pressure range from 0.05 to 1.0. The pore volumes and pore size distributions were calculated by using the Barrett-Joyner-Halenda $(\mathrm{BJH})$ model and the total pore volumes were estimated from the amount adsorbed at a relative pressure $P / P_{0}$ of 0.993 . UV-vis spectra were recorded using a Beckman DU 800 nucleic acid/protein analyser (Beckman Instruments, Inc., Rosemead, CA).

\section{Results and discussion}

Silica NPs synthesized by the Stöber method were chosen as core materials. ${ }^{13}$ Core-shell $\mathrm{SiO}_{2} @ \mathrm{Fe}_{3} \mathrm{O}_{4} / \mathrm{C}$ microspheres were first obtained in one step by simply mixing silica spheres, ferrocene, and hydrogen peroxide under hydrothermal conditions. In this reaction, ferrocene acts as an iron precursor as well as a carbon resource and hydrogen peroxide is used to oxidize ferrocene. The transmission electron microscopy (TEM) images of samples at this stage are presented in Fig. S1 (for details see $\mathrm{ESI}^{\dagger}$ ). Yolk-shell $\mathrm{SiO}_{2} @ \mathrm{Fe}_{3} \mathrm{O}_{4} / \mathrm{C}$ microspheres were obtained by selective etching of the silica core by ammonia water through another hydrothermal step. The as-prepared yolk-shell particles, with a core of $\sim 160 \mathrm{~nm}$ diameter and a shell of $\sim 30 \mathrm{~nm}$ thickness, are significantly monodisperse and highly uniform (Fig. 1a and b). The cores of the yolk-shell nanostructures are believed to be free to move, but in the dry environment of copper grid, the cores tend to adhere to the shell due to the attractive capillary force between them. In addition, the corrosion process by ammonia water did not destroy the structure of the double-layered shell, demonstrating its stability.

The size of the silica core can be easily controlled by adjusting the amount of ammonia water $\left(\mathrm{NH}_{3} \cdot \mathrm{H}_{2} \mathrm{O}, 25 \%\right)$ during the etching process. Fig. 2 demonstrates the TEM images of samples treated with various volumes of ammonia water. It is clear that the core-shell structure converted into a yolk-shell structure and the size of the silica core gradually decreased with the increasing volume of ammonia water (Fig. 2a-c). If the volume further increases, the silica cores disappear entirely which leads to the formation of hollow $\mathrm{Fe}_{3} \mathrm{O}_{4} /$ $\mathrm{C}$ nanocapsules (Fig. 2d). The $\mathrm{N}_{2}$ adsorption-desorption technique was employed to characterize the surface area, pore volume, and pore size of different nanostructures (see Fig. S2 $\dagger$ ). The Brunauer-Emmett-Teller (BET) surface areas of the coreshell structure, yolk-shell structure, and hollow structure were estimated to be $67.6 \mathrm{~m}^{2} \mathrm{~g}^{-1}, 88.1 \mathrm{~m}^{2} \mathrm{~g}^{-1}$, and $90.1 \mathrm{~m}^{2} \mathrm{~g}^{-1}$, respectively. Compared with that of the core-shell product

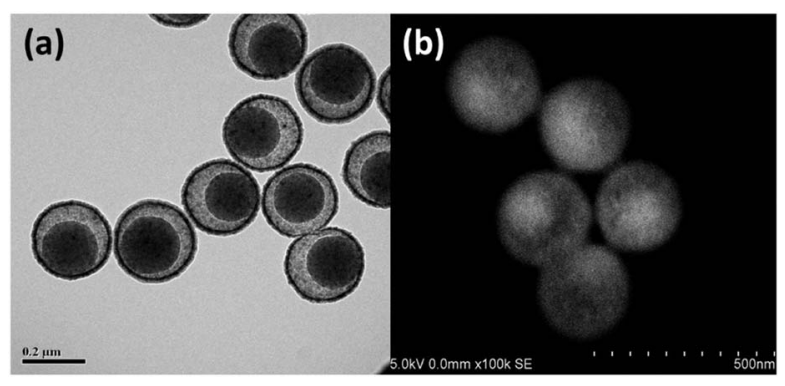

Fig. 1 TEM (a) and STEM (b) images of yolk-shell $\mathrm{SiO}_{2} @ \mathrm{Fe}_{3} \mathrm{O}_{4} / \mathrm{C}$. 


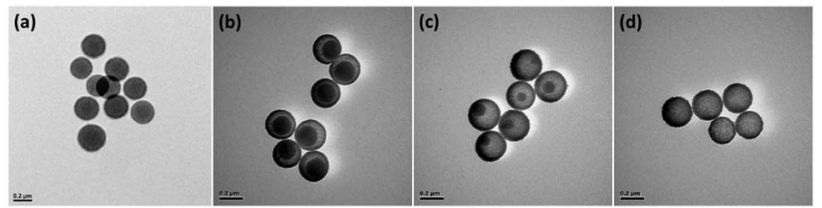

Fig. 2 TEM images of core-shell $\mathrm{SiO}_{2} @ \mathrm{Fe}_{3} \mathrm{O}_{4} / \mathrm{C}(\mathrm{a})$, yolk-shell $\mathrm{SiO}_{2} @ \mathrm{Fe}_{3} \mathrm{O}_{4} / \mathrm{C}(\mathrm{b}$ and $\mathrm{c}$ ), and hollow $\mathrm{Fe}_{3} \mathrm{O}_{4} / \mathrm{C}(\mathrm{d})$.

$\left(0.19 \mathrm{~cm}^{3} \mathrm{~g}^{-1}\right)$, the total pore volume of the yolk-shell structure and hollow capsule increase to 0.47 and $0.90 \mathrm{~cm}^{3} \mathrm{~g}^{-1}$, respectively, due to the creation of large cavities. The pore sizes of the samples calculated by the Barrett-Joyner-Halenda $(\mathrm{BJH})$ method remain almost constant $(3.4-3.8 \mathrm{~nm})$. In view of the requirement of void space to accommodate the catalytic NPs and modified silica surface to stabilize them, the yolk-shell product was eventually selected as a support for loading NPs.

Yolk-shell $\mathrm{SiO}_{2} @ \mathrm{Fe}_{3} \mathrm{O}_{4} / \mathrm{C}$ microspheres were subsequently modified with amine groups on the cavity surface and carbon shell via silanization of 3-aminopropyl-triethoxysilane (APTES).

These amine-functionalized yolk-shell microspheres would facilitate the attachment of the gold precursor $\left(\mathrm{AuCl}_{4}{ }^{-}\right)$through static interactions. After incubation with $\mathrm{HAuCl}_{4}$ aqueous solution and reduction with $\mathrm{NaBH}_{4}, \mathrm{SiO}_{2} @ \mathrm{Fe}_{3} \mathrm{O}_{4} / \mathrm{C} @ \mathrm{Au}$ nanocomposites were acquired. Fig. 3a shows a representative TEM image of the $\mathrm{SiO}_{2} @ \mathrm{Fe}_{3} \mathrm{O}_{4} / \mathrm{C} @ \mathrm{Au}$ composite microsphere which indicates that the yolk-shell architecture of the product was not affected by the deposition of Au NPs. Furthermore, plenty of Au NPs with good dispersibility are found to be distributed both on the shell and within the cavity. From the TEM image with high resolution (Fig. 3b), the tiny black dots related to Au NPs without aggregation are observed clearly to be of uniform size of $2 \mathrm{~nm}$ in diameter. To directly present the Au NPs in the interior

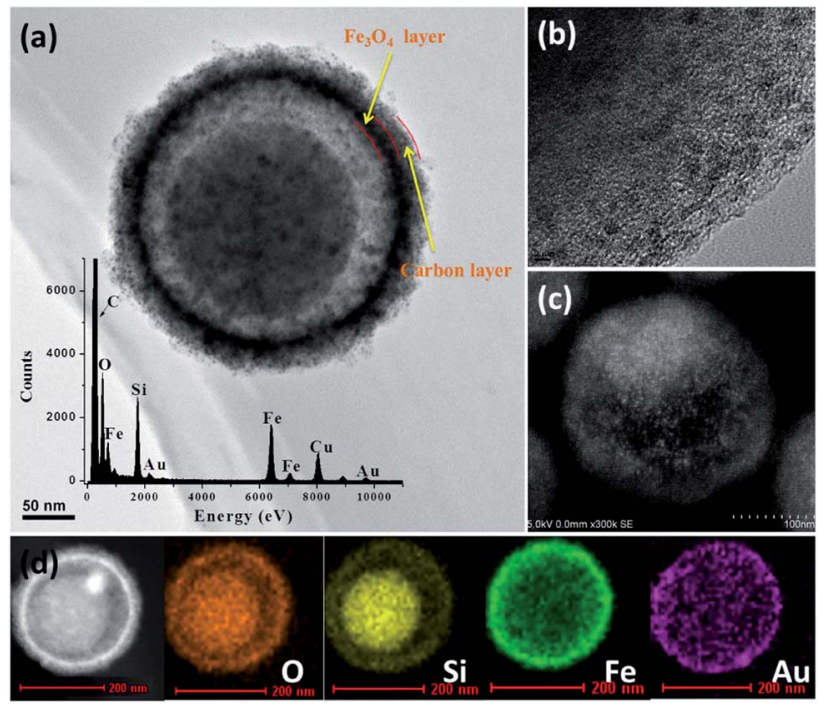

Fig. 3 (a) TEM image of yolk-shell $\mathrm{SiO}_{2} @ \mathrm{Fe}_{3} \mathrm{O}_{4} / \mathrm{C} @ \mathrm{Au}$; (b) HRTEM image of the outer shell; (c) STEM image of yolk-shell $\mathrm{SiO}_{2} @ \mathrm{Fe}_{3} \mathrm{O}_{4} / \mathrm{C} @ \mathrm{Au}$; (d) EDX mapping of a single yolk-shell $\mathrm{SiO}_{2} @ \mathrm{Fe}_{3} \mathrm{O}_{4} / \mathrm{C} @ \mathrm{Au}$ microsphere. The inset in (a) shows the EDX spectra of $\mathrm{SiO}_{2} @ \mathrm{Fe}_{3} \mathrm{O}_{4} / \mathrm{C} @ \mathrm{Au}$. area of the yolk-shell structure, scanning transmission electron microscopy (STEM) analysis was performed. The STEM image (Fig. 3c) shows that the bright dots (Au NPs) are homogeneously located on the silica surface as well as the shell, which is consistent with the TEM results. In contrast to the reported yolk-shell gold based catalyst with a sole large Au NP core in each capsule, ${ }^{2 a, 15}$ there are numerous well-dispersed Au NPs with small size $(\sim 2 \mathrm{~nm})$ encapsulated into the void space of the yolk-shell support. Moreover, the shell provides additional anchoring sites to support more Au NPs in each particle. Thereby, highly enhanced catalytic activity of the as-prepared yolk-shell catalyst can be expected because of the large number of active sites in the cavity together with the shell. The energydispersive X-ray (EDX) analyses (inset of Fig. 3a) present the elemental compositions of the nanocomposites and the involved elements $(\mathrm{C}, \mathrm{O}, \mathrm{Fe}, \mathrm{Si}$, and $\mathrm{Au}$ ) ensure the existence of iron oxide, silica oxide, and Au NPs in the yolk-shell microspheres. A more detailed elemental distribution of a single $\mathrm{SiO}_{2} @ \mathrm{Fe}_{3} \mathrm{O}_{4} / \mathrm{C} @ A$ yolk-shell particle was gained from the EDX mapping (Fig. 3d). Distinct $\mathrm{Si}$ and $\mathrm{Fe}$ signals were mainly detected from the interior $\mathrm{SiO}_{2}$ core and the $\mathrm{Fe}_{3} \mathrm{O}_{4}$ shell, respectively, while the Au signal was relatively dispersedly distributed in both the shell and the core, suggesting the immobilization of Au NPs in these areas of the yolk-shell materials.

The crystallographic structure of the product at each step was further determined by X-ray diffraction (XRD). Fig. 4a shows the XRD pattern of the core-shell $\mathrm{SiO}_{2} @ \mathrm{Fe}_{3} \mathrm{O}_{4} / \mathrm{C}$ particles. The peaks at $2 \theta=30.1^{\circ}(220), 35.4^{\circ}(311), 43.1^{\circ}(400), 57.5^{\circ}(511)$, and $62.9^{\circ}$ (440) match well with the characteristic peaks of $\mathrm{Fe}_{3} \mathrm{O}_{4}$ (JCPDS card no. 19-629) and the apparent broadening of the peaks demonstrates the nanoscaled crystals composed of an inner magnetic shell. ${ }^{16}$ The XRD pattern of yolk-shell $\mathrm{SiO}_{2}$ @ $\mathrm{Fe}_{3} \mathrm{O}_{4} / \mathrm{C}$ (Fig. 4b) exhibits similar features to the core-shell structure, indicating that the phase structure of the magnetic component was well retained after silica etching. In the pattern of $\mathrm{SiO}_{2} @ \mathrm{Fe}_{3} \mathrm{O}_{4} / \mathrm{C} @ \mathrm{Au}$, three new peaks at $2 \theta=38.2^{\circ}, 44.2^{\circ}$, and $64.8^{\circ}$ corresponding to the (111), (200), and (220) planes of $\mathrm{Au}$ are observed, confirming the presence of Au NPs. ${ }^{16}$ The size of

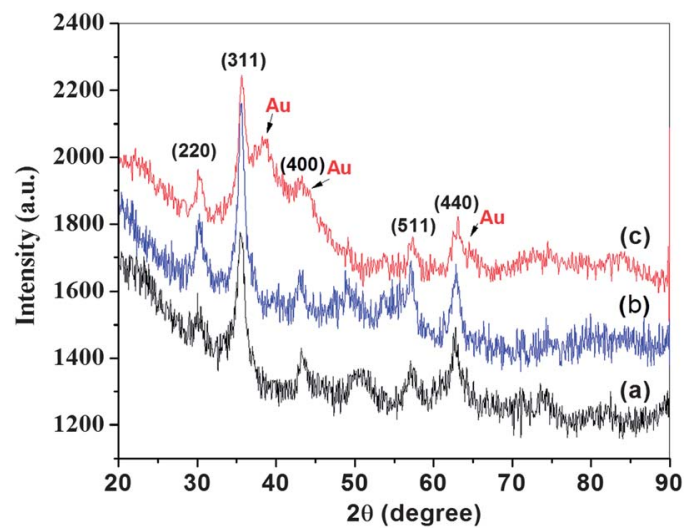

Fig. 4 XRD patterns of core-shell $\mathrm{SiO}_{2} @ \mathrm{Fe}_{3} \mathrm{O}_{4} / \mathrm{C}(\mathrm{a})$, yolk-shell $\mathrm{SiO}_{2} @ \mathrm{Fe}_{3} \mathrm{O}_{4} / \mathrm{C}$ (b), and yolk-shell $\mathrm{SiO}_{2} @ \mathrm{Fe}_{3} \mathrm{O}_{4} / \mathrm{C} @ \mathrm{Au}(\mathrm{c})$. 
the Au NPs calculated by the Scherrer formula is $3.0 \mathrm{~nm}$, which is close to the TEM observation.

Fig. 5 shows the Fourier-transform infrared (FTIR) spectra of the samples. The peaks around $1600-1800 \mathrm{~cm}^{-1}$ and 3420 $\mathrm{cm}^{-1}$ in Fig. 5a are severally assigned to $-\mathrm{C}=\mathrm{O}$ and $-\mathrm{OH}$ stretching vibrations, which means that the outer carbon shell arising from the carbonization of ferrocene at a relatively low treatment temperature $\left(210{ }^{\circ} \mathrm{C}\right)$ contains abundant carboxyl and hydroxyl groups. These oxygen-containing species help to increase the hydrophilicity and thus guarantee the water dispersivity of the nanomaterials in solution. The peaks appearing at $1080 \mathrm{~cm}^{-1}$ and $810-950 \mathrm{~cm}^{-1}$ correspond to $\mathrm{Si}-\mathrm{O}-\mathrm{Si}$ and $\mathrm{Si}-$ $\mathrm{OH}$ stretching bands, respectively. The hydroxyl groups on the silica core surface and the outer carbon shell would favor the silanization of APTES, which acts as a good anchor for capturing the gold precursor in the Au NP immobilization step. The absorption band observed at $560 \mathrm{~cm}^{-1}$ in all spectra is related to $\mathrm{Fe}-\mathrm{O}$ groups. In Fig. $5 \mathrm{c}$, the additional peak at $2910 \mathrm{~cm}^{-1}$ and the enhanced wide band around $3400 \mathrm{~cm}^{-1}$ are attributed to the $\mathrm{C}-\mathrm{H}$ stretching vibrations of propyl groups and the characteristic absorption of the $-\mathrm{NH}_{2}$ groups, respectively, which are introduced by the grafted APTES. ${ }^{17}$ After immobilization of Au NPs, the intensities of the characteristic peaks all decrease. To investigate the surface chemical composition of the product and further confirm the formation of Au NPs, the wide scan survey X-ray photo-electron spectrum (XPS) of the yolk-shell $\mathrm{SiO}_{2} @ \mathrm{Fe}_{3} \mathrm{O}_{4} / \mathrm{C} @ A u$ hybrid and high resolution spectrum of the Au element were analysed (Fig. S3 $\uparrow$ and 6). According to the survey scan spectrum, the presence of Fe 2p3 at $710.6 \mathrm{eV}$ and $\mathrm{N}$ 1s at $399.7 \mathrm{eV}$ indicates the involvement of $\mathrm{Fe}_{3} \mathrm{O}_{4}$ and the successful modification of APTES. Other elements including C 1s, O 1s, Si 2p, and Au $4 \mathrm{f}$ also exist distinctly, which are in accordance with the EDX result. The bonding energies at 84.1 and $87.8 \mathrm{eV}$ are associated with the $\mathrm{Au} 4 \mathrm{f}_{7 / 2}$ and $\mathrm{Au} 4 \mathrm{f}_{5 / 2}$ of $\mathrm{Au}^{0}$ respectively, illustrating the formation of metallic Au.

The magnetic properties of the materials at various stages were evaluated by using a vibrating sample magnetometer (VSM). Fig. S4† shows the $M-H$ hysteresis loops of the nanocomposites measured by sweeping the external field between

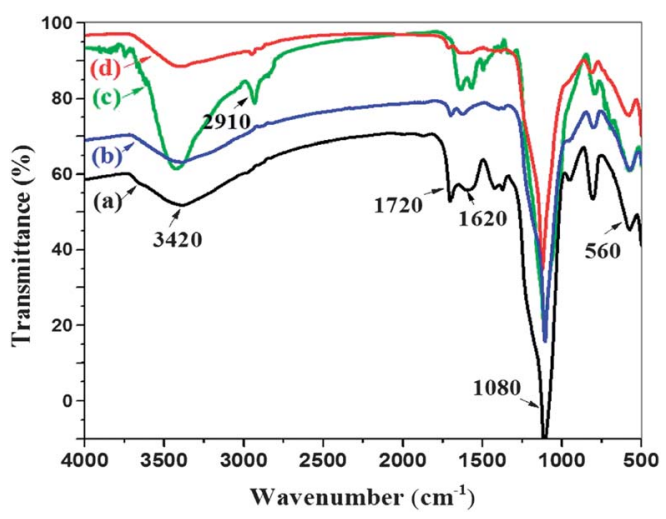

Fig. 5 FTIR spectra of core-shell $\mathrm{SiO}_{2} @ \mathrm{Fe}_{3} \mathrm{O}_{4} / \mathrm{C}(\mathrm{a})$, yolk-shell $\mathrm{SiO}_{2} @ \mathrm{Fe}_{3} \mathrm{O}_{4} / \mathrm{C}$ (b), yolk-shell $\mathrm{SiO}_{2} @ \mathrm{Fe}_{3} \mathrm{O}_{4} / \mathrm{C}$ with amine groups modified (c), and yolk-shell $\mathrm{SiO}_{2} @ \mathrm{Fe}_{3} \mathrm{O}_{4} / \mathrm{C} @ \mathrm{Au}(\mathrm{d})$.

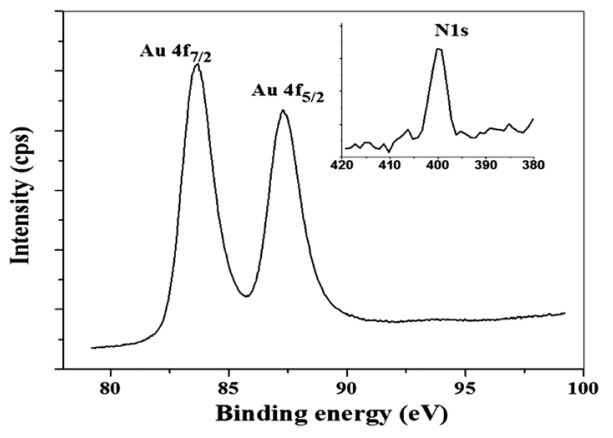

Fig. 6 Core-level XPS spectrum of Au 4f. The inset is the high resolution spectrum of $\mathrm{N} 1 \mathrm{~s}$.

-1 and $1 \mathrm{~T}$ at room temperature. The saturation magnetization value of core-shell $\mathrm{SiO}_{2} @ \mathrm{Fe}_{3} \mathrm{O}_{4} / \mathrm{C}\left(6.8 \mathrm{emu} \mathrm{g}^{-1}\right)$ is much lower than that of the yolk-shell structure $\left(28.1 \mathrm{emu}^{-1}\right)$ due to the existence of the larger non-magnetic silica core. After the loading of $\mathrm{Au}$ NPs, the saturation magnetization value of yolkshell $\mathrm{SiO}_{2} @ \mathrm{Fe}_{3} \mathrm{O}_{4} / \mathrm{C} @ \mathrm{Au}$ decreases to $19.7 \mathrm{emu} \mathrm{\textrm {g } ^ { - 1 }}$ which is still sufficient for magnetic separation from a mixture system (Fig. 8, inset). In addition, $\mathrm{N}_{2}$ adsorption-desorption experiments were also carried out (Fig. S5 $\dagger$ ) to validate the involvement of Au NPs. The BET surface area and total pore volume were decreased to $39.8 \mathrm{~m}^{2} \mathrm{~g}^{-1}$ and $0.29 \mathrm{~cm}^{3} \mathrm{~g}^{-1}$, respectively, proving the entrance of the Au NPs into the cavity and the mesoporous shell. The pore size, however, was almost unchanged, indicating the maintenance of the mesoporous shell to allow the diffusion of the reaction molecule. All the above information reveals the successful synthesis of the yolkdouble shell $\mathrm{SiO}_{2} @ \mathrm{Fe}_{3} \mathrm{O}_{4} / \mathrm{C} @ \mathrm{Au}$ composites.

The catalytic performance of the as-prepared yolk-like $\mathrm{SiO}_{2} @ \mathrm{Fe}_{3} \mathrm{O}_{4} / \mathrm{C} @ \mathrm{Au}$ nanostructures was explored in the reduction of aromatic nitrocompounds. The reduction of 4-nitrophenol to its daughter derivatives (4-aminophenol) in the presence of sodium borohydride $\left(\mathrm{NaBH}_{4}\right)$ was chosen as a model reaction (see the Experimental section) and the kinetics of 4-nitrophenol reduction was monitored by UV-vis absorption

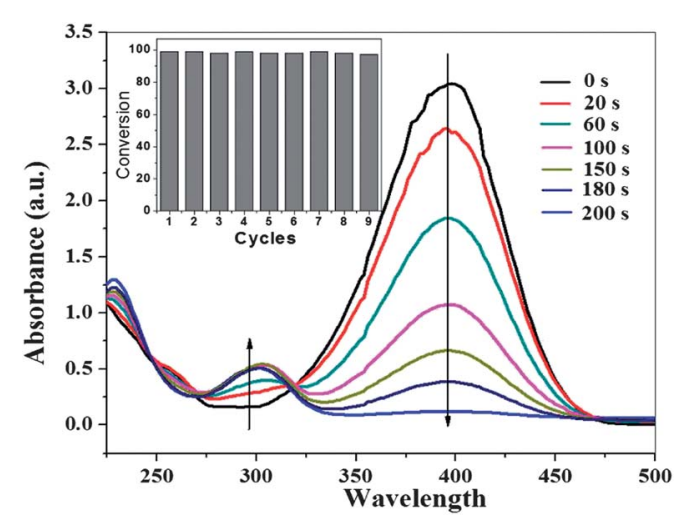

Fig. 7 Time-dependent UV-vis absorption spectra of the 4-nitrophenol reduced by $\mathrm{NaBH}_{4}$ catalyzed by yolk-shell $\mathrm{SiO}_{2} @ \mathrm{Fe}_{3} \mathrm{O}_{4} / \mathrm{C} @ \mathrm{Au}$ composites. The inset shows the conversions of 4-nitrophenol in nine successive cycles with the yolkshell $\mathrm{SiO}_{2} @ \mathrm{Fe}_{3} \mathrm{O}_{4} / \mathrm{C} @ \mathrm{Au}$ catalyst. 
spectroscopy (Fig. 7). The reaction did not proceed in the absence of catalyst, which was evidenced by a constant absorption peak at $398 \mathrm{~nm}$. After introducing the yolk-like $\mathrm{SiO}_{2} @ \mathrm{Fe}_{3} \mathrm{O}_{4} / \mathrm{C} @ \mathrm{Au}$ as the catalyst into the solution, the intensity of the characteristic absorption peak at $398 \mathrm{~nm}$ related to 4nitrophenol decreased successively, while the characteristic absorption of 4-aminophenol at $295 \mathrm{~nm}$ appeared accordingly. The peak at $398 \mathrm{~nm}$ vanished completely within $200 \mathrm{~s}$ and the reduction of 4-nitrophenol can also be visually witnessed by the discolourization of the initial yellow mixture. Moreover, the presence of an isosbestic point between the two absorption bands reveals that the catalytic reduction of 4-nitrophenol only generates 4-aminophenol without other byproducts.

Since excess $\mathrm{NaBH}_{4}$ is present and its concentration can be considered as a constant during the reaction period, the rate constant for the reduction of 4-nitrophenol permits us to assess by the pseudo-first-order kinetics. The ratios of $C_{t}$ (the concentration of 4-nitrophenol at time $t$ ) to $C_{0}$ (the initial concentration of 4-nitrophenol) were obtained from the relative intensity ratios of the respective absorbance $\left(A_{t} / A_{0}\right)$ at $398 \mathrm{~nm}$. The linear relationship of $\ln \left(C_{t} / C_{0}\right)$ versus time $(t)$ for the catalytic reaction indicates that the reduction of 4-nitrophenol follows the pseudo-first-order kinetics (Fig. S6†). The rate constant $(k)$ estimated directly from the slope of the straight line was $0.97 \mathrm{~min}^{-1}$ for the reactions catalyzed by the yolk-like $\mathrm{SiO}_{2} @ \mathrm{Fe}_{3} \mathrm{O}_{4} / \mathrm{C} @ \mathrm{Au}$ catalyst. Remarkably, the turnover frequency (TOF) is $17.4 \mathrm{~min}^{-1}$, calculated by the moles of 4-NP reduced per mole of $\mathrm{Au}$ content per consumed time under the present reaction conditions. In comparison with other similar studies, our yolk-like $\mathrm{SiO}_{2} @ \mathrm{Fe}_{3} \mathrm{O}_{4} / \mathrm{C} @ \mathrm{Au}$ catalyst exhibited a higher catalytic efficiency than those of most other catalysts for 4-nitrophenol reduction, which is evidenced by a higher TOF value in Table S1. $\uparrow$ Generally, the catalytic activity of Au NPs depends on the size, loading amount, stability, and goldsupport interaction. ${ }^{\mathbf{1 8}}$ In the present work, the smaller size (about $2 \mathrm{~nm}$ ) and larger loading amount (3.4 wt\% evaluated by ICP-MS analysis) of Au NPs should be responsible for the superior catalytic performance.

The reusability, which is an important concern for a catalyst, was investigated by recovering the yolk-like $\mathrm{SiO}_{2} @ \mathrm{Fe}_{3} \mathrm{O}_{4} / \mathrm{C} @ \mathrm{Au}$ catalyst from the reaction mixture with an external magnet and reusing it in the next round. The catalytic recycling experiments were performed under high levels of reactant and catalyst and

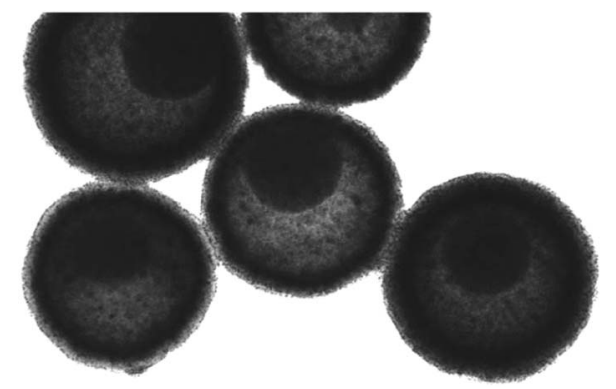

Fig. 8 TEM image of the yolk-shell $\mathrm{SiO}_{2} @ \mathrm{Fe}_{3} \mathrm{O}_{4} / \mathrm{C} @$ Au catalyst after repeated catalytic tests. the required time to thoroughly convert 4-nitrophenol to 4aminophenol at each cycle was estimated. The required time increased from $100 \mathrm{~s}$ for the 1 st cycle to $350 \mathrm{~s}$ for the 9th cycle, which implies a slight decrease in catalytic efficiency. Such a decrease may be attributed to the negligible loss of Au NPs located on the surface of the outer carbon shell as well as the adsorption of 4-aminophenol emerged from the reduction reaction onto the surface of the remaining Au NPs. ${ }^{19}$ However, the as-prepared catalyst was still highly active with a conversion over 99\% even after 9 successive cycles of reactions (the inset in Fig. 7), indicating the excellent recyclability of the catalyst. The reason is that most of the Au NPs were stably confined into the cavity and the mesoporous shell so that they were prevented from leakage. The Au content of the yolk-double shell $\mathrm{SiO}_{2} @$ $\mathrm{Fe}_{3} \mathrm{O}_{4} / \mathrm{C} @ \mathrm{Au}$ composites after nine cycles of catalysis slightly increased to $3.9 \mathrm{wt} \%$, as measured by ICP-MS, which was possibly due to the partial etching of the silica core by the high concentration of $\mathrm{NaBH}_{4} \cdot{ }^{20}$ Meanwhile, TEM observation after the repeated catalytic tests was also carried out to check the stability of the as-prepared catalyst (Fig. 8). It was found that the morphology of the yolk-double shell $\mathrm{SiO}_{2} @ \mathrm{Fe}_{3} \mathrm{O}_{4} / \mathrm{C} @ \mathrm{Au}$ microsphere was scarcely changed, suggesting the good stability of the materials.

\section{Conclusions}

In summary, a new type of yolk-like microsphere with a movable silica core, a magnetic-carbon double layered shell, and a hollow space between the core and the shell were successfully synthesized through a facile strategy. Besides the common functions in terms of providing the protection for the core and allowing the diffusion of species, the unique structure of the shell endows the composite with some new features such as superparamagnetism and ease of modification. Furthermore, a large amount of tiny Au NPs were readily immobilized within the post-functionalized interior cavity as well as the mesoporous shell by the impregnation and reduction of the $\mathrm{Au}$ precursor. The as-prepared yolk-like nanocomposites exhibit excellent catalytic performance and perfect reusability for the reduction of 4-nitrophenol. Therefore, this multi-functional nanostructure holds great promise as a novel gold-based catalyst system for many chemical reactions. Additionally, the design strategy in this work could be extended to the fabrication of other new nanosystems with enhanced properties for various advanced applications.

\section{Acknowledgements}

We thank the National Basic Research Program of China (2011CB936001) and the National Natural Science Foundation of China $(21277152,21277002)$ for financial support.

\section{Notes and references}

1 (a) R. Guttel, M. Paul and F. Schuth, Catal. Sci. Technol., 2011, 1, 65; (b) J. C. Park, H. J. Lee, H. S. Jung, M. Kim, H. J. Kim, K. H. Park and H. Song, ChemCatChem, 2011, 3, 
755; (c) C. C. Yec and H. C. Zeng, Chem. Mater., 2012, 24, 1917; (d) Y. F. Zhu, T. Ikoma, N. Hanagata and S. Kaskel, Small, 2010, 6, 471; (e) Y. Chen, Y. Gao, H. Chen, D. Zeng, Y. Li, Y. Zheng, F. Li, X. Ji, X. Wang, F. Chen, Q. He, L. Zhang and J. Shi, Adv. Funct. Mater., 2012, 22, 1586; $(f)$ T. Harada, S. Ikeda, Y. H. Ng, T. Sakata, H. Mori, T. Torimoto and M. Matsumura, Adv. Funct. Mater., 2008, 18, 2190; $(g)$ J. Liu, S. Z. Qiao, J. S. Chen, X. W. Lou, X. R. Xing and G. Q. Lu, Chem. Commun., 2011, 47, 12578; (h) T. Yang, J. Liu, Y. Zheng, M. J. Monteiro and S. Z. Qiao, Chem.-Eur. J., 2013, 19, 6942; (i) Y. Yang, X. Liu, X. B. Li, J. Zhao, S. Y. Bai, J. Liu and Q. H. Yang, Angew. Chem., Int. Ed., 2012, 51, 9164.

2 (a) J. Lee, J. C. Park and H. Song, Adv. Mater., 2008, 20, 1523; (b) S. N. Wang, M. C. Zhang and W. Q. Zhang, ACS Catal., 2011, 1, 207; (c) I. Lee, J. B. Joo, Y. D. Yin and F. Zaera, Angew. Chem., Int. Ed., 2011, 50, 10208; (d) S. Wu, J. Dzubiella, J. Kaiser, M. Drechsler, X. H. Guo, M. Ballauff and Y. Lu, Angew. Chem., Int. Ed., 2012, 51, 2229; (e) A. D. Pandey, R. Guttel, M. Leoni, F. Schuth and C. Weidenthaler, J. Phys. Chem. C, 2010, 114, 19386.

3 Q. Zhang, I. Lee, J. Ge, F. Zaera and Y. Yin, Adv. Funct. Mater., 2010, 20, 2201.

4 (a) J. Han, Y. Liu and R. Guo, Adv. Funct. Mater., 2009, 19, 1112; (b) Y. F. Wang, A. V. Biradar and T. Asefa, ChemSusChem, 2012, 5, 132; (c) J. B. Yoo, H. J. Yoo, B. W. Lim, K. H. Lee, M. H. Kim, D. Kang and N. H. Hur, ChemSusChem, 2012, 5, 2334.

5 B. Liu, W. Zhang, H. Feng and X. Yang, Chem. Commun., 2011, 47, 11727.

6 J. Liu, H. Q. Yang, F. Kleitz, Z. G. Chen, T. Yang, E. Strounina, G. Q. Lu and S. Z. Qiao, Adv. Funct. Mater., 2012, 22, 591.

7 (a) J. Lee, J. C. Park, J. U. Bang and H. Song, Chem. Mater., 2008, 20, 5839; (b) R. Liu, S. M. Mahurin, C. Li, R. R. Unocic, J. C. Idrobo, H. J. Gao, S. J. Pennycook and S. Dai, Angew. Chem., Int. Ed., 2011, 50, 6799.
8 (a) S. H. Wu, C. T. Tseng, Y. S. Lin, C. H. Lin, Y. Hung and C. Y. Mou, J. Mater. Chem., 2011, 21, 789; (b) F. Dong, W. Guo, S. K. Park and C. S. Ha, Chem. Commun., 2012, 48, 1108; (c) S. Wu, J. Dzubiella, J. Kaiser, M. Drechsler, X. Guo, M. Ballauff and Y. Lu, Angew. Chem., Int. Ed., 2012, 51, 2229; (d) Z. Jin, F. Wang, F. Wang, J. Wang, J. C. Yu and J. Wang, Adv. Funct. Mater., 2013, 23, 2137.

9 (a) S. Shylesh, V. Schunemann and W. R. Thiel, Angew. Chem., Int. Ed., 2010, 49, 3428; (b) L. Aschwanden, B. Panella, P. Rossbach, B. Keller and A. Baiker, ChemCatChem, 2009, 1, 111; (c) D. Rosario-Amorin, X. Wang, M. Gaboyard, R. Clerac, S. Nlate and K. Heuze, Chem.-Eur. J., 2009, 15, 12636.

10 (a) Q. Fang, S. Xuan, W. Jiang and X. Gong, Adv. Funct. Mater., 2011, 21, 1902; (b) W. R. Zhao, H. R. Chen, Y. S. Li, L. Li, M. D. Lang and J. L. Shi, Adv. Funct. Mater., 2008, 18, 2780 .

11 T. Zeng, X. L. Zhang, Y. R. Ma, S. H. Wang, H. Y. Niu and Y. Q. Cai, Chem. Commun., 2013, 49, 6039.

12 H. Wu, S. Zhang, J. Zhang, G. Liu, J. Shi, L. Zhang, X. Cui, M. Ruan, Q. He and W. Bu, Adv. Funct. Mater., 2011, 21, 1850.

13 W. Stober, A. Fink and E. Bohn, J. Colloid Interface Sci., 1968, 26, 62.

14 K. Cheng, Y.-M. Zhou, Z.-Y. Sun, H.-B. Hu, H. Zhong, X.-K. Kong and Q.-W. Chen, Dalton Trans., 2012, 41, 5854.

15 J. Liu, S. Z. Qiao, S. Budi Hartono and G. Q. Lu, Angew. Chem., Int. Ed., 2010, 122, 5101.

16 T. Zeng, X. L. Zhang, Y. R. Ma, H. Y. Niu and Y. Q. Cai, J. Mater. Chem., 2012, 22, 18658.

17 M. Z. Kassaee, H. Masrouri and F. Movahedi, Appl. Catal., A, 2011, 395, 28.

18 L. F. Chen, J. C. Hu and R. Richards, J. Am. Chem. Soc., 2009, 131, 914.

19 F. H. Lin and R. A. Doong, J. Phys. Chem. C, 2011, 115, 6591.

20 T. R. Zhang, J. P. Ge, Y. X. Hu, Q. Zhang, S. Aloni and Y. D. Yin, Angew. Chem., Int. Ed., 2008, 47, 5806. 